\title{
Modeling the Pulse Rate, Respiratory Rate, and Weight of Congestive Heart Failure Patients: A Case Study at Wachemo University Nigist Eleni Mohammed Memorial Referral Hospital
}

\author{
Mohammed Sultan ( $\sim$ sultankarebo@gmail.com ) \\ Wachemo University \\ Ritbano Ahmed Abdo \\ Wachemo University
}

\section{Research Article}

Keywords: Acute Coronary failure, Congestive heart failure, Intra-class Correlation Coefficient, Marginal Quasi-Likelihood

Posted Date: July 16th, 2021

DOl: https://doi.org/10.21203/rs.3.rs-567998/v1

License: (c) (1) This work is licensed under a Creative Commons Attribution 4.0 International License. Read Full License 


\section{Abstract}

Background: The linear mixed model is one of the common models used to analyze the longitudinal data; it may comprise of Separate (Univariate), joint Bivariate or joint multivariate linear mixed model, which is predicated on the number of response variables incorporated in the analysis. Adjusting for correlation matrix and covariance matrix between and within subjects is one reason why modern longitudinal data analysis techniques are deemed more appropriate than some of the previous methods of analysis. Some studies assume that the correlation between observations is zero. However, it is unlikely that repeated measurements on the same individual will actually be independent. To that end, comparing the different linear mixed models and identifying the appropriate model demonstrates the evolution of patients with CHF.

Methods: In this study the separate, bivariate and multivariate linear mixed models were analyzed with different covariance and correlation structures. The parameters in the models were estimated by maximum likelihood estimation and restricted maximum likelihood estimation techniques. The models were compared by AIC, BIC, and Log-likelihood ratio test.

Results: The models with unstructured covariance structure for random effects and autoregressive order one for serial correlation structure had small AIC, BIC and -2LL and standard errors. Separate models had high AIC, BIC and -2LL and standard errors than bivariate and multivariate had small AIC, BIC and -2LL and standard errors than all models.

Conclusions: Finally, a multivariate linear mixed model with autoregressive order one correlation structure and unstructured covariance structure for random effects, to consider within and between patients' variations, was considered as the best model to depict the evolution of patients with congestive heart failure.

\section{Background}

In longitudinal data, the dependent variable is measured several times, whereas the individual subject is used as a random factor. More specifically, Linear mixed models (LMMs) or General Linear mixed models (GLMM) can be used to account for repeated measures in longitudinal studies as well as for random effects [1].

Mixed models are commonly used to tackle correlated data or hierarchical data in the fields of health and social sciences [1]. In this context, the linear mixed model is one of the mixed models that fit the mean response as a combination of population characteristics (fixed effects) that are assumed to be shared by all individuals and subject-specific effects (random effects) unique to a particular individual [2].

Depending on the number of response variables incorporated into the analysis, there are different types of linear mixed models: separate (univariate), bivariate and multivariate linear mixed models. 
The separate (univariate) linear mixed model clarifies the relationship between a continuous response variable and the independent variables, with both fixed and random effects. Conversely, the bivariate or multivariate linear mixed model describes the relationship between two or more continuous response variables and the independent variables respectively [3]. In most longitudinal experiments, the number of outcomes measured repeatedly in the subjects is found to exceed one. However, whenever the purpose is a comparison of longitudinal trends between outcomes, or the purpose is to identify the association between the outcomes and how that association evolves over time, a joint analysis of all outcomes is required [4].

In a different study, analysing the multiple outcomes jointly is considered the best model. For example, multivariate linear mixed models were proposed to analyse multiple outcomes as well as to assess and test for a global exposure effect across outcomes, while assuming a flexible correlation structure for the multiple outcomes [5]. A study conducted in Spain revealed that the multivariate approach performs better than the univariate approach [6]. In other studies conducted in France, the bivariate random effects model was significantly better than two separate univariate random effects models $[7,8]$.

The correlation matrix and/or covariance matrix between observations also plays an important role in the analysis of longitudinal data. Adjusting for correlation between observations is one reason why modern longitudinal data analysis techniques are more appropriate than some of the previous analysis methods [9]. Correlation structures are used to model dependency among within-group errors [10,11]. Some studies assume that the correlation between measurement errors amounts to zero [12]. This assumption may not hold in some data situations, especially if the time between observations is very short (i.e. daily or weekly observations) or if the correlation between observations does not decrease very quickly [13]. It is unlikely that repeated measurements on the same individual will actually be independent [9]. Ignoring the existing correlation of longitudinal data may lead to incorrect and inefficient inferences, and it may increase the risk of Type I errors and of underestimating standard errors [9]. Consideration of all these issues is necessary in order to select an appropriate model with the mean, covariance and correlation structure for the longitudinal data of Congestive Heart Failure patients (CHF) patients. The main aim of this study was identifying the appropriate linear mixed model demonstrates the evolution of patients with CHF.

\section{Methods}

\section{Source of data and Variables}

The current study's data comprises 154 adult patients suffering from congestive heart failure, who reported that they had visited a hospital at least thrice within two years. The patients' pulse rate, respiratory rate and weight were considered as response variables, while age, sex, time, place of residence, New York Heart Association Class, diagnostic history, left ventricle ejection fraction, valvular heart disease, smoking status, diabetes status, diastolic blood pressure and systolic blood pressure were deemed as independent variables. 


\section{Statistical Methods of Data Analysis}

Statistical analysis contain both descriptive and inferential statistics, such as: summary statistics, Exploratory Data Analysis and model comparison have been used in this study. Joint random effects with LMM have been modeled to infer the joint effect of multivariate longitudinal outcomes of CHF patients.

\section{Separated linear mixed effect model}

The model which used in this study was a linear mixed effect model which contains fixed effects, random effects, serial correlation and measurement errors and are referred as $\beta, b_{i}, \varepsilon_{1 i}$ and $\varepsilon_{2 i}$ respectively.

The three endpoints are longitudinally measured as a vector of responses, $Y_{i}(t)$ at each occasion. Let

$Y_{i}=\left[\begin{array}{c}Y_{k i}(t) \\ Y_{j i}(t) \\ Y_{l i}(t)\end{array}\right]$, the response vectors for the patient i with $Y_{\mathrm{ki}}, Y_{\mathrm{ji}}$, and $Y_{\mathrm{li}}$, the $Y_{\mathrm{ki}}, Y_{\mathrm{ji}}$, and $Y_{\mathrm{li}}$ is vector of the end points for $\mathrm{k}, \mathrm{j}, \mathrm{I}(\mathrm{k}, \mathrm{j}, \mathrm{I}=1,2,3)$ with $n_{1 i}=n_{2 i}=n_{3 i}=n_{i}$. In generally linear mixed model is written in the form of:

$$
Y_{i}(t)=X_{i}(t)^{T} \beta+Z_{i}(t)^{T} b_{i}+\epsilon_{1 i}(t)+\epsilon_{2 i}(t)-----(1)
$$

For each of response variables it can be rewritten in the form of

$$
Y_{k i}(t)=\mu_{k}(\mathrm{t})+\alpha_{k i}+b_{k i}(t)+\epsilon_{1 k i}(t)+\epsilon_{2 k i}(t)-----(2)
$$

Where $Y_{i}(t)$ : Measurement of response in $i^{\text {th }}$ patient at time $t, X_{i}(t)$ : Vector of fixed covariate for $i^{\text {th }}$ patient at time $t, Z_{i}(t)$ : Vector of random covariate for $i^{\text {th }}$ patient at time $t, \beta$ : Vector of unknown parameters associated with fixed covariate (of dimension $\mathrm{k}$ ), The $b_{i}$ is a Vector of unknown parameters associated with random covariate for $i^{\text {th }}$ patient and $\sim N(0, D)$. It is assumed to be independent of measurement error and serial correlation.

The $n_{i} X 1$ vector $\epsilon_{1 i}$ represents serial correlation, the correlation of sub-sequent measurements within a patient. It is assumed that $\epsilon_{1 i}$ has a normal distribution with mean zero and covariance matrix $H_{i}$ distributed independently of measurement error. The $n_{i} x 1$ vector $\epsilon_{2 i}$ stands for the measurement error part, the components of $\epsilon_{2 i}$ are assumed uncorrelated and normally distributed with mean zero and common variance $\sigma^{2}$.

\section{Joint linear mixed effect model}

The joint model is extension of separate (univariate) models which used to study the joint evolution of two or more biomarkers. In this study the joint which investigated was bivariate and multivariate linear 
mixed effect model. For the pairs $(k, j)$ of response variables the bivariate linear mixed effect model is given as:

$$
\begin{aligned}
& \left.\begin{array}{c}
Y_{k i}(t)=\mu_{k}(\mathrm{t})+\alpha_{k i}+b_{k i}(t)+\epsilon_{1 k i}(t)+\epsilon_{2 k i}(t) \\
Y_{j i}(t)=\mu_{j}(\mathrm{t})+\alpha_{j i}+b_{j i}(t)+\epsilon_{1 j i}(t)+\epsilon_{2 j i}(t)
\end{array}\right\}------(3) \\
& , \mathrm{b}=\left(\begin{array}{l}
\alpha_{i} \\
b_{i}
\end{array}\right)=\left(\begin{array}{l}
\left(\begin{array}{c}
\alpha_{k i} \\
\alpha_{j i}
\end{array}\right) \\
\left(\begin{array}{c}
b_{k i} \\
b_{j i}
\end{array}\right)
\end{array}\right) \sim \mathrm{N}\left(0, D_{4 \times 4}\right),
\end{aligned}
$$

where $D$ is the variance-covariance matrix for the 2 intercepts and 2 slopes associated with every outcome (covariance matrix for random effects), ( $\left.\varepsilon_{1 k i}(\mathrm{t}), \varepsilon_{1 j i}(\mathrm{t})\right) \sim$ $\mathrm{N}(0,2 \times 2)$, Where $2 \times 2$ is matrix of serial correlation structures, $J$, Kforallj $\neq k$,

$$
\left(\varepsilon_{2 k i}(\mathrm{t}), \varepsilon_{2 j i}(\mathrm{t})\right) \tilde{N}\left(0 \quad\left[\begin{array}{cc}
\sigma_{k}^{2} & 0 \\
0 & \sigma_{j}^{2}
\end{array}\right]\right) \text {. }
$$

The multivariate linear mixed effect model is given as:

$$
\left.\begin{array}{c}
Y_{k i}(t)=\mu_{k}(\mathrm{t})+\alpha_{k i}+b_{k i}(t)+\epsilon_{1 k i}(t)+\epsilon_{2 k i}(t) \\
Y_{j i}(t)=\mu_{j}(\mathrm{t})+\alpha_{j i}+b_{j i}(t)+\epsilon_{1 j i}(t)+\epsilon_{2 j i}(t) \\
Y_{l i}(t)=\mu_{l}(\mathrm{t})+\alpha_{l i}+b_{l i}(t)+\epsilon_{1 l i}(t)+\epsilon_{2 l i}(t)
\end{array}\right\}
$$

$$
, \mathrm{b}=\left(\begin{array}{c}
\alpha_{i} \\
b_{i}
\end{array}\right)=\left(\begin{array}{c}
\left(\begin{array}{c}
\alpha_{k i} \\
a_{j i} \\
\alpha_{l i}
\end{array}\right) \\
\left(\begin{array}{c}
b_{k i} \\
b_{j i} \\
b_{l i}
\end{array}\right)
\end{array}\right), \sim \mathrm{N}\left(0, D_{6 x 6}\right)
$$

where $D$ is the variance-covariance matrix for the 3 intercepts and 3 slopes associated with every outcome (covariance matrix for random effects), $\left(\varepsilon_{1 k i}(\mathrm{t}), \varepsilon_{1 j i}(\mathrm{t}), \varepsilon_{1 l i}(\mathrm{t})\right) \sim \mathrm{N}(0,3 X 3)$, Where $3 X 3$ is matrix of serial correlation structures, $J$, $K$, andlforallj $\neq k \neq l$,

$$
\left(\varepsilon_{2 k i}(\mathrm{t}), \varepsilon_{2 j i}(\mathrm{t}), \varepsilon_{2 l i}(\mathrm{t})\right) \tilde{N}\left(0 \quad\left[\begin{array}{ccc}
\sigma_{k}^{2} & 0 & 0 \\
0 & \sigma_{j}^{2} & 0 \\
0 & 0 & \sigma^{2}{ }_{l}
\end{array}\right]\right)
$$


In the linear mixed model setup the covariance structure of the repeated measurements can be viewed as consisting of two components that; the structure induced by the random coefficient model, which is typically non-stationary and the residual components which accounts for deviations of the observations about the individual profiles. Most of the time there has been a tendency to use a very simple structure for component (residual components), often independent deviations with common variance. This has implied that the random coefficient model should capture most of the structure of the variance and covariance of the repeated measurements. In this study the researchers relaxed this assumption (independent deviations with common variance) by including the variance-covariance structure for random effects and serial correlation structure to accounts for deviations of the observations about the individual profiles separately. The variance-covariance and correlation matrixes used in this study were listed in the Table 1. 
Table 1

The Variance-Covariance/Correlation Structures

\begin{tabular}{|c|c|c|}
\hline Type & Matrix R & $\begin{array}{l}\text { Number of } \\
\text { Parameters }\end{array}$ \\
\hline Unstructured (UN) & $\mathrm{UN}=\left[\begin{array}{cccc}\sigma_{1}{ }^{2} & \sigma_{12} & \ldots & \sigma_{1 i} \\
\sigma_{21} & \sigma_{2}^{2} & \ldots & \sigma_{2 i} \\
: & : & : & : \\
\sigma_{i 1} & \sigma_{i 2} & \ldots & \sigma_{i}^{2}\end{array}\right]$ & $i(i+1) / 2$ \\
\hline Compound Symmetry (CS) & $\mathrm{CS}=\sigma^{2}\left[\begin{array}{cccc}1 & \rho & \ldots & \rho \\
& 1 & \ldots & \vdots \\
& & & \ddots \\
& & & 1\end{array}\right]$ & 2 \\
\hline $\begin{array}{l}\text { Heterogeneous Compound symmetry } \\
\text { (CSH) }\end{array}$ & $\mathrm{CSH}=\left[\begin{array}{cccc}\sigma_{1}{ }^{2} & \sigma_{1} \sigma_{2} \rho & \ldots \sigma_{1} \sigma_{i} \rho \\
& \sigma_{2}{ }^{2} & \ldots & \sigma_{2} \sigma_{i} \rho \\
& & \ddots & \vdots \\
& & & \sigma_{i}^{2}\end{array}\right.$ & $i+1$ \\
\hline First Order Autoregressive (AR(1)) & $\operatorname{AR}(1)=\sigma^{2}\left[\begin{array}{cccc}1 & \rho & \ldots \rho^{i-1} \\
& 1 & \ldots \rho^{i-2} \\
& & \ddots & \vdots \\
& & & 1\end{array}\right]$ & 2 \\
\hline Toeplitz (TOEP) & $\begin{array}{l}\text { TOEP }= \\
\left.\qquad \begin{array}{ccccc}\sigma^{2} & \sigma_{12} & & & \\
& \sigma_{13} \ldots & \sigma_{1 i} \\
& \sigma^{2} & \sigma_{12} \ldots & \sigma_{1 i-1} \\
& & \sigma^{2} & \cdots & \vdots \\
& & & \ddots & \sigma_{12} \\
& & & & \sigma^{2}\end{array}\right]\end{array}$ & $\mathrm{T}$ \\
\hline
\end{tabular}




\begin{tabular}{|llll|}
\hline Type & Matrix R & & $\begin{array}{l}\text { Number of } \\
\text { Parameters }\end{array}$ \\
\hline Variance Components $(\mathrm{VC})$ & $\mathrm{VC}=\left[\begin{array}{cccc}\sigma_{1}^{2} & 0 & \ldots 0 \\
0 & \sigma_{2}^{2} & \ldots 0 \\
: & : & : & : \\
0 & 0 & \ldots & \sigma_{i}^{2}\end{array}\right]$ \\
\hline
\end{tabular}

\section{Parameter Estimation}

To estimate the parameters pertaining to the model used, both maximum likelihood estimation (MLE) and restricted maximum likelihood estimation were performed. The ML method primarily maximizes the log likelihood with respect to the variance parameters, while treating the fixed effects parameters $\beta$ as constant. After determining the variance parameter estimates, the fixed effects parameters are identified by ascertaining the values of $\beta$, which maximize the log likelihood, while treating the variance parameters as constant. Consequently, the variance parameters are estimated by maximizing the REML log likelihood with regard to the variance. Given the nature of the REML likelihood and its treatment of fixed effects as parameters rather than as constants, the resulting variance parameter estimates can be considered as unbiased. Moreover, it reduces the bias inherent in the ML estimates of covariance parameters [14].

\section{Model Comparisons}

In the case of repeated or longitudinal data where selecting the best model means not only to select the best mean structure but also the most optimal variance covariance structure for model. In this study the most commonly known model selection criteria which are Akaike Information Criterion (AIC), the Bayesian Information Criterion (BIC), and Log-likelihood ratio test were used to select the best model.

\section{Results}

\section{Source of data and Variables}

The current study's data comprises 154 adult patients suffering from congestive heart failure, who reported that they had visited a hospital at least thrice within two years. The patients' pulse rate, respiratory rate and weight were considered as response variables, while age, sex, time, place of residence, New York Heart Association Class, diagnostic history, left ventricle ejection fraction, valvular heart disease, smoking status, diabetes status, diastolic blood pressure and systolic blood pressure were deemed as independent variables.

\section{Statistical Methods of Data Analysis}


Statistical analysis contain both descriptive and inferential statistics, such as: summary statistics, Exploratory Data Analysis and model comparison have been used in this study. Joint random effects with LMM have been modeled to infer the joint effect of multivariate longitudinal outcomes of CHF patients.

\section{Separated linear mixed effect model}

The model which used in this study was a linear mixed effect model which contains fixed effects, random effects, serial correlation and measurement errors and are referred as $\beta, b_{i}, \varepsilon_{1 i}$ and $\varepsilon_{2 i}$ respectively.

The three endpoints are longitudinally measured as a vector of responses, $Y_{i}(t)$ at each occasion. Let

$Y_{i}=\left[\begin{array}{c}Y_{k i}(t) \\ Y_{j i}(t) \\ Y_{l i}(t)\end{array}\right]$, the response vectors for the patient i with $Y_{\mathrm{ki}}, Y_{\mathrm{ji}}$, and $Y_{\mathrm{li}}$, the $Y_{\mathrm{ki}}, Y_{\mathrm{ji}}$, and $Y_{\mathrm{li}}$ is vector

of the end points for $\mathrm{k}, \mathrm{j}, \mathrm{I}(\mathrm{k}, \mathrm{j}, \mathrm{I}=1,2,3)$ with $n_{1 i}=n_{2 i}=n_{3 i}=n_{i}$. In generally linear mixed model is written in the form of:

$$
Y_{i}(t)=X_{i}(t)^{T} \beta+Z_{i}(t)^{T} b_{i}+\epsilon_{1 i}(t)+\epsilon_{2 i}(t)-----(1)
$$

For each of response variables it can be rewritten in the form of

$$
Y_{k i}(t)=\mu_{k}(\mathrm{t})+\alpha_{k i}+b_{k i}(t)+\epsilon_{1 k i}(t)+\epsilon_{2 k i}(t)-----(2)
$$

Where $Y_{i}(t)$ : Measurement of response in $i^{\text {th }}$ patient at time $t, X_{i}(t)$ : Vector of fixed covariate for $i^{\text {th }}$ patient at time $t, Z_{i}(t):$ Vector of random covariate for $i^{t h}$ patient at time $t, \beta$ : Vector of unknown parameters associated with fixed covariate (of dimension $\mathrm{k}$ ), The $b_{i}$ is a Vector of unknown parameters associated with random covariate for $i^{\text {th }}$ patient and $\sim N(0, D)$. It is assumed to be independent of measurement error and serial correlation.

The $n_{i} x 1$ vector $\epsilon_{1 i}$ represents serial correlation, the correlation of sub-sequent measurements within a patient. It is assumed that $\epsilon_{1 i}$ has a normal distribution with mean zero and covariance matrix $H_{i}$ distributed independently of measurement error. The $n_{i} x 1$ vector $\epsilon_{2 i}$ stands for the measurement error part, the components of $\epsilon_{2 i}$ are assumed uncorrelated and normally distributed with mean zero and common variance $\sigma^{2}$.

\section{Joint linear mixed effect model}

The joint model is extension of separate (univariate) models which used to study the joint evolution of two or more biomarkers. In this study the joint which investigated was bivariate and multivariate linear mixed effect model. For the pairs $(k, j)$ of response variables the bivariate linear mixed effect model is given as: 


$$
\left.\begin{array}{c}
Y_{k i}(t)=\mu_{k}(\mathrm{t})+\alpha_{k i}+b_{k i}(t)+\epsilon_{1 k i}(t)+\epsilon_{2 k i}(t) \\
Y_{j i}(t)=\mu_{j}(\mathrm{t})+\alpha_{j i}+b_{j i}(t)+\epsilon_{1 j i}(t)+\epsilon_{2 j i}(t)
\end{array}\right\}------
$$

, $\mathrm{b}=\left(\begin{array}{c}\alpha_{i} \\ b_{i}\end{array}\right)=\left(\begin{array}{c}\left(\begin{array}{c}\alpha_{k i} \\ \alpha_{j i}\end{array}\right) \\ \left(\begin{array}{c}b_{k i} \\ b_{j i}\end{array}\right)\end{array}\right) \tilde{N}\left(0, D_{4 \times 4}\right)$, where D is the variance-covariance matrix for the 2

intercepts and 2 slopes associated with every outcome (covariance matrix for random effects), $\left(\varepsilon_{1 k i}(\mathrm{t}), \varepsilon_{1 j i}(\mathrm{t})\right) \sim \mathrm{N}(0,2 X 2)$, Where $2 X 2$ is matrix of serial correlation structures, $J$, Kforallj $\neq k$, $\left(\varepsilon_{2 k i}(\mathrm{t}), \varepsilon_{2 j i}(\mathrm{t})\right) \tilde{N}\left(0 \quad\left[\begin{array}{cc}\sigma_{k}^{2} & 0 \\ 0 & \sigma_{j}^{2}\end{array}\right]\right)$.

The multivariate linear mixed effect model is given as:

$$
\left.\begin{array}{c}
Y_{k i}(t)=\mu_{k}(\mathrm{t})+\alpha_{k i}+b_{k i}(t)+\epsilon_{1 k i}(t)+\epsilon_{2 k i}(t) \\
Y_{j i}(t)=\mu_{j}(\mathrm{t})+\alpha_{j i}+b_{j i}(t)+\epsilon_{1 j i}(t)+\epsilon_{2 j i}(t) \\
Y_{l i}(t)=\mu_{l}(\mathrm{t})+\alpha_{l i}+b_{l i}(t)+\epsilon_{1 l i}(t)+\epsilon_{2 l i}(t)
\end{array}\right\}
$$

, $\mathrm{b}=\left(\begin{array}{c}\alpha_{i} \\ b_{i}\end{array}\right)=\left(\begin{array}{l}\left(\begin{array}{c} \\ \alpha_{k i} \\ \alpha_{j i} \\ \alpha_{l i}\end{array}\right) \\ \left(\begin{array}{c} \\ b_{k i} \\ b_{j i} \\ b_{l i}\end{array}\right)\end{array}\right)$, (tilde $\backslash \operatorname{text}\{\mathrm{N}\}\left(0,\{\mathrm{D}\}_{-}\{6 \mathrm{x} 6\}\right)$, where $\mathrm{D}$ is the variance-covariance

matrix for the 3 intercepts and 3 slopes associated with every outcome (covariance matrix for random effects), $\left(\varepsilon_{1 k i}(\mathrm{t}), \varepsilon_{1 j i}(\mathrm{t}), \varepsilon_{1 l i}(\mathrm{t})\right) \sim \mathrm{N}(0,3 \times 3)$, Where $3 X 3$ is matrix of serial correlation structures, $J$, $K$, andlforallj $\neq k \neq l$, 


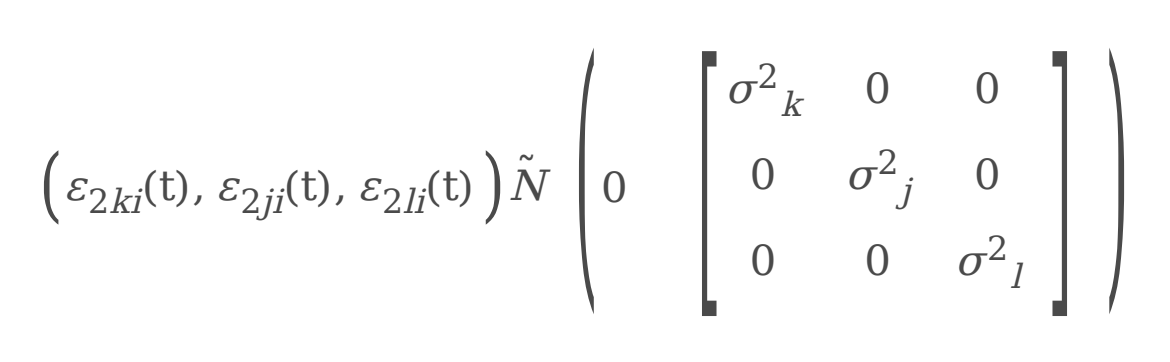

\section{Variance-Covariance and Correlation Structures}

In the linear mixed model setup the covariance structure of the repeated measurements can be viewed as consisting of two components that; the structure induced by the random coefficient model, which is typically non-stationary and the residual components which accounts for deviations of the observations about the individual profiles. Most of the time there has been a tendency to use a very simple structure for component (residual components), often independent deviations with common variance. This has implied that the random coefficient model should capture most of the structure of the variance and covariance of the repeated measurements. In this study the researchers relaxed this assumption (independent deviations with common variance) by including the variance-covariance structure for random effects and serial correlation structure to accounts for deviations of the observations about the individual profiles separately. The variance-covariance and correlation matrixes used in this study were listed in the Table 1. 
Table 1

The Variance-Covariance/Correlation Structures

\begin{tabular}{|c|c|c|}
\hline Type & Matrix R & $\begin{array}{l}\text { Number of } \\
\text { Parameters }\end{array}$ \\
\hline Unstructured (UN) & $\mathrm{UN}=\left[\begin{array}{cccc}\sigma_{1}{ }^{2} & \sigma_{12} & \ldots & \sigma_{1 i} \\
\sigma_{21} & \sigma_{2}^{2} & \ldots & \sigma_{2 i} \\
: & : & : & : \\
\sigma_{i 1} & \sigma_{i 2} & \ldots & \sigma_{i}^{2}\end{array}\right]$ & $i(i+1) / 2$ \\
\hline Compound Symmetry (CS) & $\mathrm{CS}=\sigma^{2}\left[\begin{array}{cccc}1 & \rho & \ldots & \rho \\
& 1 & \ldots & \vdots \\
& & & \ddots \\
& & & 1\end{array}\right]$ & 2 \\
\hline $\begin{array}{l}\text { Heterogeneous Compound symmetry } \\
\text { (CSH) }\end{array}$ & $\mathrm{CSH}=\left[\begin{array}{cccc}\sigma_{1}{ }^{2} & \sigma_{1} \sigma_{2} \rho & \ldots \sigma_{1} \sigma_{i} \rho \\
& \sigma_{2}{ }^{2} & \ldots & \sigma_{2} \sigma_{i} \rho \\
& & \ddots & \vdots \\
& & & \sigma_{i}^{2}\end{array}\right.$ & $i+1$ \\
\hline First Order Autoregressive (AR(1)) & $\operatorname{AR}(1)=\sigma^{2}\left[\begin{array}{cccc}1 & \rho & \ldots \rho^{i-1} \\
& 1 & \ldots \rho^{i-2} \\
& & \ddots & \vdots \\
& & & 1\end{array}\right]$ & 2 \\
\hline Toeplitz (TOEP) & $\begin{array}{l}\text { TOEP }= \\
\left.\qquad \begin{array}{ccccc}\sigma^{2} & \sigma_{12} & & & \\
& \sigma_{13} \ldots & \sigma_{1 i} \\
& \sigma^{2} & \sigma_{12} \ldots & \sigma_{1 i-1} \\
& & \sigma^{2} & \cdots & \vdots \\
& & & \ddots & \sigma_{12} \\
& & & & \sigma^{2}\end{array}\right]\end{array}$ & $\mathrm{T}$ \\
\hline
\end{tabular}




\begin{tabular}{|c|c|c|c|c|}
\hline Type & Matr & & & $\begin{array}{l}\text { Number of } \\
\text { Parameters }\end{array}$ \\
\hline Variance Components (VC) & $\mathrm{VC}=$ & $\begin{array}{cc}\sigma_{1}^{2} & 0 \\
0 & \sigma_{2}^{2} \\
: & : \\
0 & 0\end{array}$ & 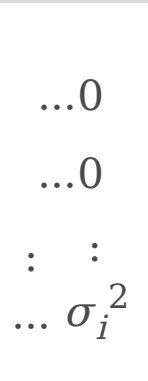 & Q \\
\hline
\end{tabular}

\section{Parameter Estimation}

To estimate the parameters pertaining to the model used, both maximum likelihood estimation (MLE) and restricted maximum likelihood estimation were performed. The ML method primarily maximizes the log likelihood with respect to the variance parameters, while treating the fixed effects parameters $\beta$ as constant. After determining the variance parameter estimates, the fixed effects parameters are identified by ascertaining the values of $\beta$, which maximize the log likelihood, while treating the variance parameters as constant. Consequently, the variance parameters are estimated by maximizing the REML log likelihood with regard to the variance. Given the nature of the REML likelihood and its treatment of fixed effects as parameters rather than as constants, the resulting variance parameter estimates can be considered as unbiased. Moreover, it reduces the bias inherent in the ML estimates of covariance parameters [14].

\section{Model Comparisons}

In the case of repeated or longitudinal data where selecting the best model means not only to select the best mean structure but also the most optimal variance covariance structure for model. In this study the most commonly known model selection criteria which are Akaike Information Criterion (AIC), the Bayesian Information Criterion (BIC), and Log-likelihood ratio test were used to select the best model.

\section{Discussion}

In this study three models were considered for fitting three outcome variables of congestive heart failure patients which measured longitudinally. They were separate, bivariate and multivariate linear mixed effects models. The models contain the fixed and random effect components. The appropriate fixed effects and random effects were selected by using the maximum likelihood estimation method and restricted maximum likelihood estimation method respectively. The linear mixed model without random effects, with only intercept, with intercept and linear time, and with intercept and quadratic time were fitted. The fitted random effect models were compared for the purpose of selecting the best random effects that enable to account the variability between congestive heart failure patients as $[12,15]$. The four random effect models were compared using the AIC, BIC and - 2LL value. Though a model with random effects intercept and linear time was the best after transformation of time for a log of pulse rate and $\log$ of respiratory rate in to the square root of time and the response variable pulse rate, respiratory 
rate and weight into logarithmic function which contradict with [12] in which time was linear and the distributions were normal without transformation.

The covariance structures of random effects which used in this study were; unstructured, compound symmetry, heterogeneous compound symmetry, Toeplitz, variance components and autoregressive covariance structure of order one. In similar to the study $[7,12,15$, and 16] the unstructured covariance structure was the most appropriate covariance structure of the models from the random effects of separation, bivariate and multivariate models.

In order to model dependence among observations the serial correlation structure was considered. The correlation structures which used in this study were compound symmetric; variance components and autoregressive correlation structure of order one and compared to select the most appropriate one by using AIC, BIC and - 2LL. The autoregressive structure of order one was the most appropriate correlation structure as $[17,18]$ incorporated into mixed models to consider the dependence among the observations which contradict the independence assumptions in $[12,15,16]$. The congestive heart failure is chronic disease which does not recover in short time. The measurement of the outcome of the congestive heart failure patients does not decrease very quickly. Taking the autoregressive structure of order one correlation structure is appropriate as [13] stated that; the assumption of independent correlation may not hold if the observation does not decrease very quickly.

Finally, with the selected covariance structure of random effects and serial correlation structure, the three models (separate, bivariate and multivariate models) were compared by using AIC, BIC and - 2LL. In addition to these tests there were high standard errors of parameters in the separate model than bivariate model than multivariate model (supplementary table A, B, and C). The multivariate model is better than bivariate model is better than separate model. It shows that for congestive heart failure data joint model is better than separate as $[7,12,15$, and 19$]$.

\section{Conclusion}

For congestive heart failure data the random effect model is better with unstructured covariance structure of random effects and an Auto-regressive order one serial correlation structure. Some studies consider the only covariance structure of random effects, in addition to the covariance structure of random effects considering the correlation structure of repeated effects needed for congestive heart failure data. Though, using independent correlation structures of measurement error with-out considering serial correlation structure to model dependency within observations may display less accurate results.

Three models considered in this study were separate, bivariate and multivariate for fitting three response variables measured longitudinally. The multivariate model with an unstructured covariance structure of random effects and an Auto-regressive order one serial correlation structure is the best model compared to the separate and joint bivariate models because its standard error of the parameter estimates is smaller. And also, the multivariate model has a very small AIC value which indicates that it fits the data better than the separate and joint bivariate models. 
In this study, it focuses on only three response variables with serial correlation structure of compound symmetry, variance components and autoregressive order one, for future work, one might want to look at modeling more than three response variables over time with an additional serial correlation structure.

\section{Abbreviations}

ACF

Acute Coronary failure, AIC:Akaike Information Criterion, AU:Africa Union, BIC:Bayesian Information Criterion, CAD:Coronary artery disease, CHD:Coronary heart disease, CHF:Congestive heart failure, GEE:Generalized estimating equations, GLM:Generalized Linear Model, Hr:Heart rate, HF:Heart failure, ICC:Intra-class Correlation Coefficient, LMM:Linear mixed model, LVEF:Left-ventricular ejection fraction, MLIRT:Multilevel item response theory, MLMM:Multivariate linear mixed model, MQL:Marginal QuasiLikelihood, NYHAC:New York heart association class, PQL:Penalized Quasi-Likelihood, Pr:Pulse rate, Rr:Respiration rate, SBP:Systolic blood pressure, DBP:Diastolic blood pressure, Sqrtim:square root of time, Wh:Weight

\section{Declarations}

\section{Authors' contributions}

MS conceptualized the idea conceive, designed, wrote the study, participated in data collection, analyzed the data, interpreted the result, participated in the drafting the manuscript and approved the final manuscript for submission, responsible for submission and communication about the manuscript. RA, participated in the drafting the manuscript, revised the manuscript and approved the final manuscript for submission.

\section{Funding}

This research was funded by the Jimma University. The funding body had no role in the design or analysis of the study, interpretation of results, or writing of the manuscript.

\section{Availability of data and materials}

All relevant data are within the manuscript and its supporting information files.

\section{Ethics approval and consent to participate}

The ethics approval was obtained from Jimma University review board (IRB). Additionally, permission was obtained from the hospital authority before beginning the data collection from the patients card.

\section{Consent for publication}

Not applicable. 


\section{Competing interests}

The authors declare that they have no competing interests.

\section{Acknowledgements}

We extend our gratitude to Jimma University, Hospital authorities, supervisors, data collectors, and respondents who participated on this study.

\section{References}

1. Wang KS. Linear and Non-Linear Mixed Models in Longitudinal Studies and Complex Survey Data. J Biom Biostat. 2016; 7: 290. doi:10.4172/2155-6180.1000290.

2. Yende N. Modelling CD4 + Count Over Time in HIV Positive Patients Initiated on HAART in South Africa Using Linear Mixed Models. MSC. Thesis, the University of KwaZulu Natal, December 2009. Available at: https://pdfs.semanticscholar.org/db70/3bf91673ce2782f09d52f776542550f0e40f.pdf

3. Ene M, Leighton EA, Blue GL, Bell BA. Multilevel Models for Categorical Data Using SAS ${ }^{\circ} P R O C$ : The Basics. Paper 3430 - 2015, 2015.

4. Verbeke G, Fieuws S, Molenberghs G, Davidian M. The analysis of multivariate longitudinal data: a review. Stat Methods Med Res. 2014; 23(1): 42-59.

5. Sammel M, Lin X, Ryan L. Multivariate linear mixed models for multiple outcomes. Stat Med. 1999; 18:2479-2492.

6. Santos AA, Nogales FJ, Ruiz E. Comparing univariate and multivariate models to forecast portfolio value-at-risk. Journal of financial econometrics. 2013; 11(2):400-41.

7. Thiébaut R, Jacqmin-Gadda H, Chêne G, Leport C, Commenges D. Bivariate Linear Mixed Models Using SAS Proc MIXED, Comput Methods Programs Biomed. 2002;69(3):249-56.

8. Badiru AB. Computational survey of univariate and multivariate learning curve models. Engineering Management, IEEE Transactions on. 1992; 39(2):176-88.

9. Santos AA, Nogales FJ, Ruiz E. Comparing univariate and multivariate models to forecast portfolio value-at-risk. Journal of financial econometrics. 2013; 11(2):400-41.

10. Edwards LJ. Modern statistical techniques for the analysis of longitudinal data in biomedical research. Pediatric Pulmonology. 2000; 30(4):330-344.

11. Pinheiro JC and Bates DM. Mixed-Effects Models in S and S-PLUS. Springer Verlag New York, 2000; 96.

12. Olkin, I. and Tate, R. Multivariate correlation models with mixed discrete and continuous variables. Ann of Math Stat. 1961; 32, 448-465.

13. Fissuh and Muleta. A Joint Model for a Longitudinal Pulse Rate and Respiratory Rate of Congestive Heart Failure Patients: at Ayder Referral Hospital of Mekelle University, Tigray, Ethiopia. J Biom Biostat. 2015; 6:5.,DOI: 10.4172/2155-6180.1000260. 
14. Browne W, Goldstein H. MCMC sampling for multilevel model with non-independent residuals within and between cluster units. Journal of Educational and Behavioral Statistics. 2010; 35(4), 453-473. Doi: $10.3102 / 1076998609359788$.

15. Morrell, C.H. Likelihood ratio testing of variance components in the linear mixed-effects model using restricted maximum likelihood. Biometrics 1998; 54, 1560.

16. Negash Y, Kassahunb W, Gurmessaa A and Birliea B. Joint modeling of longitudinal systolic and diastolic blood pressure measurements of hyper-tensive patients receiving treatment. Electronic Journal of Applied Statistical Analysis EJASA, Electron. J. App. Stat. Anal 2016; 09(02): 308-325.

17. Demeke L, Dereje T, Haile M. Bivariate longitudinal data analysis: a case of hypertensive patients at Felege Hiwot Referral Hospital, Bahir Dar, Ethiopia. BMC Res. 2017; 10:722.

18. He B and Luo S. Joint modeling of multivariate longitudinal measurements and survival data with application to Parkinson's disease. Stat Methods Med Res. 2016; 25(4): 1346-1358. doi:10.1177/0962280213480877.

19. Chi, E. M. and Reinsel, G. C. Models for longitudinal data with random effects and AR (1) errors. Journal of the American Statistical Association. 1989; <background-color:\#FFCC66;bverticalalign:super;>84</background-color:\#FFCC66;bvertical-align:super;>: 452-459. 56.

20. Lindstrom, M. J. and Bates, D. M. Nonlinear mixed effects models for repeated measures data. Biometrics. 1990; <background-color:\#FFCC66;bvertical-align:super;>46</backgroundcolor:\#FFCC66;bvertical-align:super;>: 673-687.

\section{Supplementary Files}

This is a list of supplementary files associated with this preprint. Click to download.

- suportivetableandfigure.docx 